\title{
Optimization of the finite element model of Corrugated Cardboard based on an orthotropic material constitutive model
}

\author{
Fei Song ${ }^{1, a}$, Changli Liü ${ }^{1, b}$ and Guoxing Zhu ${ }^{1, c}$ \\ ${ }^{1}$ School of Mechanical and Power Engineering, East China University of Science and Technology, \\ Shanghai 200237, China \\ aflysong110@163.com, bclliu@ecust.edu.cn, ${ }^{c}$ zgx920531@163.com
}

Keywords: corrugated cardboards, finite element method, orthotropic material constitutive model

Abstract. This paper presents a study on improvement of the finite element model of corrugated cardboards. There is one shortcoming in modeling the stiffness of such cardboards in literature: neglect of the material non-linear effect of cardboards and cannot predict the peak load. A finite element method was applied to overcome this shortcoming - particularly by adopting an orthotropic material constitutive model.

\section{Introduction}

The corrugated cardboard is widely used in packaging industry because it is cost-effective, easy for recycling, and environment-friendly [1]. A typical corrugated cardboard is illustrated in Fig. 1, which is made up of paper and has a sandwich structure, in particular consisting of a corrugated core (flute) and two liners [2].

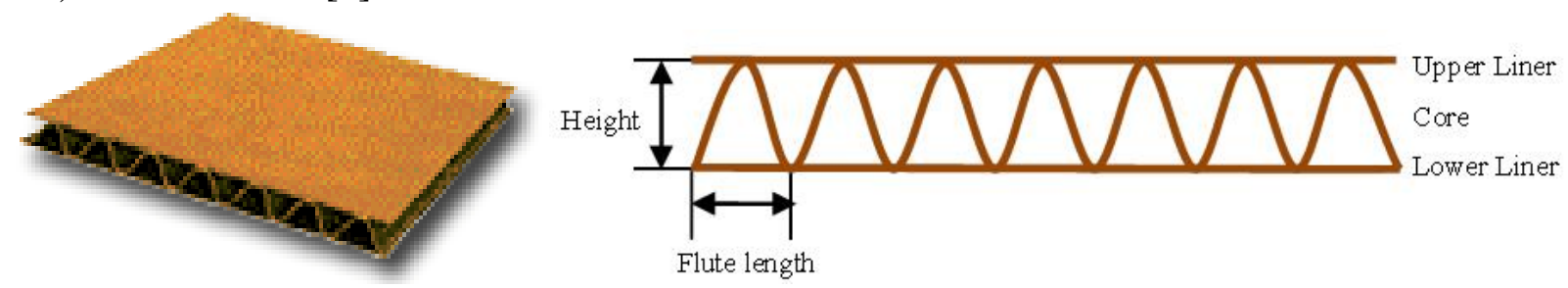

Fig. 1 A corrugated cardboard

There are several studies on modeling and prediction of the stiffness of corrugated cardboards under compressive loading in its thickness direction and several finite element models were proposed. $\mathrm{Lu}$ et al. [3] proposed a finite element model to predict the compressive behavior of the corrugated cardboard under a uniform flat compressive loading. They used the elasto-plastic material which was represented by a bi-linear constitutive model following the J2-flow theory [3]. They discovered that the result differs from the experimental result significantly with an error of about $30 \%$. They concluded that the error is caused by the use of inaccurate non-linear constitutive relation for the material used. Krusper et al. [4] refined the Lu's model with a more accurate model. In their model, a linear elastic material property was however assumed. Their model predicted result and experimental result match until the peak load (displacement is $0.2 \mathrm{~mm}$ in this case) was reached. They found that the peak load was governed by the buckling principle [4]. However, the relation between the first peak of the load and displacement failed to be accurately predicted by their model, because the model did not consider the nonlinear material property.

The study in this paper developed an accurate finite element model by adopting an orthotropic material constitutive model to capturing the information of the peak load. The experiment was conducted to verify the model. The study was with no consideration of the humidity and the thermal effect on the cardboard from the environment. 


\section{Orthotropic material constitutive model}

In this paper, an orthotropic material constitutive model was employed for the two liners and core of the corrugated cardboard. The orthotropic constitutive model consists of two parts: the linear elastic and the nonlinear plastic portions. The linear elastic portion is governed by orthotropic Hooke`s Law, while the plastic portion is governed by a quadratic Hill yield criterion.

\section{Linear elastic material property.}

The linear elastic orthotropic constitutive model is represented by [5]

$$
\left[\begin{array}{c}
\varepsilon_{x} \\
\varepsilon_{y} \\
\varepsilon_{z} \\
\gamma_{x y} \\
\gamma_{x z} \\
\lambda_{y z}
\end{array}\right]=\left[\begin{array}{cccccc}
\frac{1}{E_{x}} & \frac{-v_{y x}}{E_{y}} & \frac{-v_{z x}}{E_{z}} & 0 & 0 & 0 \\
\frac{-v_{x y}}{E_{x}} & \frac{1}{E_{y}} & \frac{-v_{z y}}{E_{z}} & 0 & 0 & 0 \\
\frac{-v_{x z}}{E_{x}} & \frac{-v_{y z}}{E_{y}} & \frac{1}{E_{z}} & 0 & 0 & 0 \\
0 & 0 & 0 & \frac{1}{G_{x y}} & 0 & 0 \\
0 & 0 & 0 & 0 & \frac{1}{G_{x z}} & 0 \\
0 & 0 & 0 & 0 & 0 & \frac{1}{G_{y z}}
\end{array}\right] \times\left[\begin{array}{c}
\sigma_{x} \\
\sigma_{x} \\
\sigma_{x} \\
\tau_{x y} \\
\tau_{x z} \\
\tau_{y z}
\end{array}\right]
$$

Where $\varepsilon_{x}, \varepsilon_{x}, \varepsilon_{x}$ is Strains in the $\mathrm{x}, \mathrm{y}, \mathrm{z}$ direction, $\gamma_{x y}, \gamma_{x z}, \gamma_{y z}$ is Strains in the $\mathrm{xy}, \mathrm{xz}, \mathrm{yz}$ plane, $E_{x}, E_{y}, E_{z}$ is Young`s modulus in the $\mathrm{x}, \mathrm{y}, \mathrm{z}$ direction, $v_{x y}, v_{x z}, v_{y z}$ is Poisson ratio in the $\mathrm{xy}, \mathrm{xz}, \mathrm{yz}$ plane and $G_{x y}, G_{x z}, G_{y z}$ is Shear modulus in the xy, xz, yz plane.

The symmetrical geometry of the cardboard leads to [5]:

$$
\frac{v_{x y}}{E_{x}}=\frac{v_{y x}}{E_{y}}, \frac{v_{x z}}{E_{x}}=\frac{v_{y x}}{E_{z}}, \frac{v_{y z}}{E_{y}}=\frac{v_{z y}}{E_{z}}
$$

Thus, there are nine unknown parameters to be determined, and they are: $E_{x}, E_{y}, E_{z}, v_{x y}, v_{x z}, v_{y z}, G_{x y}, G_{x z}, G_{y z}$.Generally, all these parameters have to be measured. However, it is impossible to measure some of the parameters due to the small dimension in the thickness direction of the liner and core. For the cardboard system as shown in Fig. 1, the in-plane material parameters $\left(E_{x}\right.$ and $E_{y}$ ) was measured by the standard tensile test, while $E_{z}, G_{x y}, G_{x z}, G_{y z}$ were derived empirically as follows. For the Young's modulus in the thickness direction, it was approximated given by

$$
E_{z}=E_{x} / 200
$$

The shear modulus are approximated by

$$
G_{x y}=0.387 \sqrt{E_{x} E_{y}}, G_{x z}=E_{x} / 55, G_{y z}=E_{y} / 55
$$

For both the liner and core, the value of $v_{x y}, v_{x z}$ and $v_{y z}$ were set according to Nordstand [6], which is $0.34,0.01$ and 0.01 , respectively. A trial and error procedure was performed, in which different values of the material parameters were tested in order to get the results as close as possible to that of the experiments. Based on this trial and error procedure, the elastic material parameters used for the model are listed in Table 1.

\section{Nonlinear plastic material property.}

In this study, Quadratic Hill yield criterion in ANSYS was used, as the model was simplified by assuming there is no difference in yield strength in tension and compression. The yield criterion was used with the isotropic hardening option, which is given by Eq. (5) from [7].

$$
f\{\sigma\}=\sqrt{\{\sigma\}^{T}[M]\{\sigma\}}-\sigma_{0}\left(\varepsilon^{p}\right)=0
$$


where $\sigma_{0}$ is yield stress in the $\mathrm{x}$ direction, $\varepsilon_{p}$ is equivalent plastic strain, $\{\sigma\}$ is yield stress matrix and

$[M]$ is plastic compliance matrix.

The plastic compliance matrix $[M]$ can be written as [7]

$$
M=\left[\begin{array}{cccccc}
G+H & -H & -G & 0 & 0 & 0 \\
-H & F+H & -F & 0 & 0 & 0 \\
-G & -F & F+G & 0 & 0 & 0 \\
0 & 0 & 0 & 2 N & 0 & 0 \\
0 & 0 & 0 & 0 & 2 L & 0 \\
0 & 0 & 0 & 0 & 0 & 2 M
\end{array}\right]
$$

where F, G, H, L, M and $\mathrm{N}$ are material constants that can be determined experimentally. They were defined by [7]

$$
\begin{gathered}
F=\frac{1}{2}\left(\frac{1}{R_{y y}^{2}}+\frac{1}{R_{z z}^{2}}-\frac{1}{R_{x x}^{2}}\right), G=\frac{1}{2}\left(\frac{1}{R_{z z}^{2}}+\frac{1}{R_{x x}^{2}}-\frac{1}{R_{y y}^{2}}\right), H=\frac{1}{2}\left(\frac{1}{R_{x x}^{2}}+\frac{1}{R_{y y}^{2}}-\frac{1}{R_{z z}^{2}}\right) \\
L=\frac{3}{2}\left(\frac{1}{R_{y z}^{2}}\right), M=\frac{3}{2}\left(\frac{1}{R_{x z}^{2}}\right), N=\frac{3}{2}\left(\frac{1}{R_{x y}^{2}}\right)
\end{gathered}
$$

In the above equations, the yield stress ratios $R_{x x}, R_{y y}, R_{z z}, R_{x y}, R_{y z}$ and $R_{x z}$ can be found by [7]

$$
R_{x x}=\frac{\sigma_{x x}^{y}}{\sigma_{0}}, R_{y y}=\frac{\sigma_{y y}^{y}}{\sigma_{0}}, R_{z z}=\frac{\sigma_{z z}^{y}}{\sigma_{0}}, R_{x y}=\sqrt{3} \frac{\sigma_{x y}^{y}}{\sigma_{0}}, R_{y z}=\sqrt{3} \frac{\sigma_{y z}^{y}}{\sigma_{0}}, R_{x z}=\sqrt{3} \frac{\sigma_{x z}^{y}}{\sigma_{0}}
$$

where $\sigma_{i j}^{y}$ is the yield stress in the $\mathrm{x}, \mathrm{y}, \mathrm{z}, \mathrm{xy}, \mathrm{yz}$ and $\mathrm{xz}$ direction. Further, the plastic slope of the material after yield point is given by

$$
E^{p l}=\frac{E_{x} E_{t}}{E_{x}-E_{t}}
$$

where $E_{x}$ is lastic modulus in the $\mathrm{x}$ direction and $E_{t}$ is tangent modulus after the yield point.

In the above equations, we need to determine $\sigma_{0}, E_{t}, R_{x x}, R_{y y}, R_{z z}, R_{x y}, R_{y z}$ and $R_{x z}$. Specifically, the in-plane material parameters $\sigma_{0}, E_{t}, R_{x x}, R_{y y}$ were derived from the results of tensile testing, while the values of $R_{z z}, R_{x y}, R_{y z}, R_{x z}$ are related to the values of $\sigma_{z z}^{y}, \sigma_{x y}^{y}, \sigma_{y z}^{y}, \sigma_{x z}^{y}$, respectively, which are the yield stress in z, xy, yz and xz direction, respectively. According to [8], $\sigma_{z z}^{y}$ was about 0.003-0.007 Gpa, while $\sigma_{x y}^{y}, \sigma_{y z}^{y}, \sigma_{x z}^{y}$ are about 0.003 to $0.011 \mathrm{Gpa}$. In this work, for the liner and core, $\sigma_{z z}^{y}$ was set to be $0.007 \mathrm{Gpa}$ and $0.004 \mathrm{Gpa}$, and $\sigma_{x y}^{y}, \sigma_{y z}^{y}, \sigma_{x z}^{y}$ were all set to be 0.011 and 0.004 for the first estimate, respectively. Further, $R_{z z}, R_{x y}, R_{y z}, R_{x z}$ were determined according to Eqn.(8). A trial and error procedure was performed, in which different values of the material parameters were tested in order to get the results as close as possible to that of the experiment. Based on this trial and error procedure, the plastic material parameters are listed in Table 1. 
Table1 Plastic and Elastic material parameters of the liner and core of the corrugated board

\begin{tabular}{|c|c|c|c|c|c|}
\hline $\begin{array}{c}\text { Plastic material } \\
\text { property }\end{array}$ & Liner & Core & $\begin{array}{c}\text { Elastic material } \\
\text { property }\end{array}$ & Liner & Core \\
\hline$\sigma_{0}(G p a)$ & 0.030 & 0.011 & $E_{x}(G p a)$ & 3.200 & 5.000 \\
\hline$E_{t}(G p a)$ & 2.500 & 0.010 & $E_{y}(G p a)$ & 2.000 & 1.300 \\
\hline$R_{x x}$ & 1.000 & 1.000 & $E_{z}(G p a)$ & 0.016 & 0.025 \\
\hline$R_{y y}$ & 0.350 & 0.300 & $v_{x y}$ & 0.340 & 0.340 \\
\hline$R_{z z}$ & 0.230 & 0.300 & $v_{y z}$ & 0.010 & 0.010 \\
\hline$R_{x y}$ & 0.635 & 0.630 & $v_{x z}$ & 0.010 & 0.010 \\
\hline$R_{y z}$ & 0.635 & 0.630 & $G_{x y}(G p a)$ & 1.000 & 1.000 \\
\hline$R_{x z}$ & 0.635 & 0.630 & $G_{y z}(G p a)$ & 0.058 & 0.050 \\
\hline & & & $G_{x z}(G p a)$ & 0.057 & 0.005 \\
\hline
\end{tabular}

\section{Results and discussions}

A typical finite element result for a $C$ flute corrugated cardboard with length $30.4 \mathrm{~mm}$ and width $30.4 \mathrm{~mm}$ is shown in Fig.3, where the horizontal axis is the prescribed displacement and the vertical axis is the force. Upon loading, the specimen responds linearly up to the first peak load (at $0.8 \mathrm{~mm}$ ). After the peak load, a decrease of force can be observed from the Fig. 2.

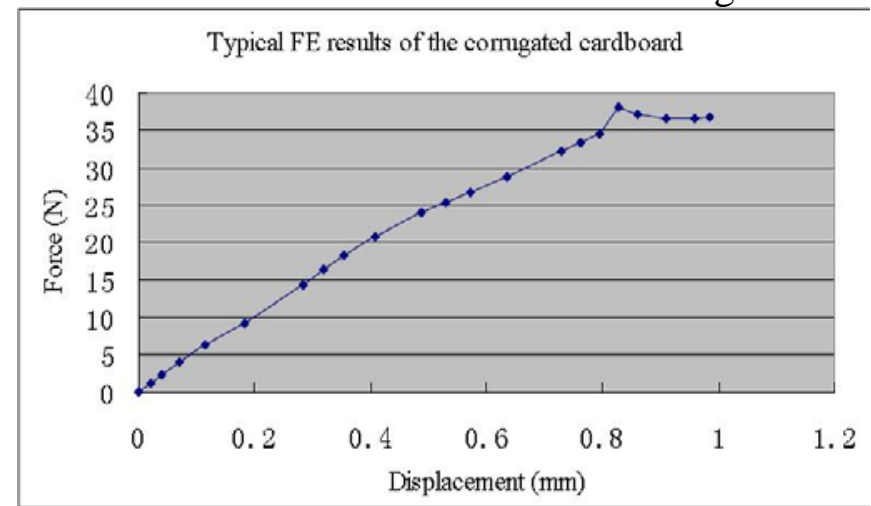

Fig. 2 Typical FE model result $(30.4 \mathrm{~mm} \times 30.4 \mathrm{~mm})$

\section{Conclusions}

This paper presented a study of the improvement of the finite element model of the corrugated cardboard. A improved finite element model of the corrugated cardboard with a nonlinear orthotropic material constitutive model was presented in ANSYS environment. In particular, the peak load of the corrugated cardboard can be predicted.

\section{Acknowledgments}

The authors are grateful to the China Natural Science Funds (NSFC, Grant No. 51175179), and the Fundamental Research Funds for the Central Universities for providing financial support for this work.

\section{References}

[1] Talbi, N., Batti, A., Ayad, R., Guo, Y.Q. , 2009, An analytical homogenization model for finite element modeling of corrugated cardboard, Composite Structure, vol. 88, no. 2, pp. 280-9.

[2] Campell, A. C., 2010, The use of A-flute, B-flute, AC flute and BC flute corrugated paperboard as a cushion material, Master`s thesis, Clemson University.

[3] Lu, T. J., and Chen, C., 2001, Compressive behaviour of corrugated board panels, Journal of Composite Materials, vol. 35, no. 23, pp. 2098-2126. 
[4] Krusper, A., Isaksson, P., and Gradin, P., 2007, Modeling of Out-of-Plane Compression Loading of Corrugated Paper Board Structures, J. Eng. Mech., vol. 133, no.11, pp. 1171-1177.

[5] Allansson, A., and Svard, B., 2001, Stability and collapse of corrugated board; numerical and experimental analysis, Master`s Thesis, Sweden: Division of Structural Mechanics, LTH, Lund University.

[6] Nordstrand, T., Carlsson, L. A., 1997, Evaluation of transverse shear stiffness of structural core sandwich plates, Composite Structure, vol. 37, pp. 145-153.

[7] ANSYS, 2004. ANSYS Release 8.1 Documentation Preview, Swanson Analysis System, Inc., Houston.

[8] Gooren, L.G.J., 2006, Creasing Behavior of Corrugated Board - An Experimentaand Numerical Approach, Department Mechanical Engineering, Report MT06.06, Eindhoven/The Netherlands. 